\title{
Confounding Factors in Exposure-Response Analyses and Mitigation Strategies for Monoclonal Antibodies in Oncology
}

\author{
Sonoko Kawakatsu ${ }^{1}$, Rene Bruno ${ }^{1}$, Matts Kågedal ${ }^{1}$, Chunze Li ${ }^{1}$, Sandhya Girish ${ }^{1}$, Amita \\ $\mathrm{Joshi}^{1}$, and Benjamin $\mathrm{Wu}^{1}$ \\ ${ }^{1}$ Genentech Inc
}

August 20, 2020

\begin{abstract}
Dose selection and optimization is an important topic in drug development to maximize treatment benefits for all patients. While exposure-response (E-R) analysis is a useful method to inform dose-selection strategy, in oncology, special considerations for prognostic factors are needed due to their potential to confound the E-R analysis for monoclonal antibodies. The current review focuses on three different approaches to mitigate the confounding effects for monoclonal antibodies in oncology: (1) coxproportional hazards modeling and case-matching, (2) tumor growth inhibition-overall survival (TGI-OS) modeling, and (3) multiple dose level study design. In the presence of confounding effects, studying multiple dose levels may be required to reveal the true E-R relationship. However, it is impractical for pivotal trials in oncology drug development programs. Therefore, the strengths and weaknesses of the other two approaches are considered, and the favorable utility of TGI-OS modeling to address confounding in E-R analyses is described. In the broader scope of oncology drug development, this review discusses the downfall of the current emphasis on E-R analyses using data from single dose level trials, and proposes that development programs be designed to study more dose levels in earlier trials.
\end{abstract}

\section{INTRODUCTION}

While contemporary drug development in oncology strives to deliver novel therapies to patients rapidly, it is also important to optimize dosing regimens to improve patient-centered care. Doses selected for pivotal trials may be efficacious doses, but not necessarily optimal to minimizing toxicity and maximizing clinical efficacy for all patients. Exposure-response (E-R) analysis is an approach that is used to support dose selection by characterizing the relationship between drug concentrations, efficacy, and safety. A variety of E-R analyses have supported dose labeling of many approved oncology drugs. ${ }^{1}$ Among the oncology therapies, however, additional complexity has been observed in characterizing E-R relationships for monoclonal antibodies. Specifically, prognostic factors can impact both pharmacokinetics (PK) and efficacy. This may result in a correlation between exposure and outcome that does not represent a causal E-R relationship and therefore, may not provide a useful basis for dose recommendations. This was exemplified by the HELOISE trial (NCT01450696) of trastuzumab, which was conducted as part of a post-marketing requirement. Following the phase 3 trial ToGA (NCT01041404), trastuzumab was approved in combination with chemotherapy for first-line treatment of HER2-positive advanced gastric cancer. An E-R analysis, however, found that the patients in the lowest exposure quartile had an overall survival (OS) approximately 8 months shorter than those with higher exposures. ${ }^{2}$ This suggested that trastuzumab exposure in this low-exposure subgroup may survival benefit, and supported the requirement of conducting a post-marketing trial for a higher dose. For this requirement, in the HELOISE trial, a higher trastuzumab dose was compared with the labeled dose in a population with similar prognostic factors as the low-exposure subgroup of the ToGA trial. Despite reliably increasing exposure, the higher dose did not improve OS in patients. ${ }^{3}$ This discrepancy between the results of the E-R analysis and the HELOISE trial indicates confounding in E-R analyses of monoclonal antibodies 
at a single dose level in oncology.

In addition to the potential confounding factors for E-R analyses in oncology, there have been reports of time-dependent changes in the PK that require additional considerations. Monoclonal antibodies that target B-cell receptors, such as rituximab, have been reported to exhibit time-dependent decrease in clearance (CL) owing to target mediated drug disposition (TMDD). ${ }^{4-6}$ Time-dependent PK has also been observed for checkpoint inhibitors nivolumab, pembrolizumab, durvalumab, and avelumab. Across these molecules the range of CL decrease over time was $17 \%$ to $32 \% \cdot{ }^{7-10}$ Best overall response was included as a covariate on CL in the time-dependent population PK models of nivolumab and pembrolizumab. ${ }^{7}, 9$ The time-dependent population PK model of durvalumab included time-varying albumin and tumor size as covariates on CL. ${ }^{8}$ While the model for avelumab did not incorporate response or time-varying biomarkers as covariates on CL, visual inspection of change in CL over time demonstrated a larger reduction in CL in responders than in nonresponders. ${ }^{10}$ Overall, the decrease in CL over time in these molecules corresponded to changes in patients' prognoses based on their responses to treatments over time. This observation may be attributed to changes in catabolic degradation of the monoclonal antibodies as a result of changing disease status. ${ }^{11}$ The changing drug CL and patient prognostic factors over time could potentially confound E-R analyses.

In this review we will discuss key considerations in interpreting E-R relationships and mitigation strategies to address the confounding effects in E-R analyses in oncology.

\section{EXPOSURE-RESPONSE (E-R) ANALYSIS CONSIDERATIONS IN ONCOLOGY}

In order to address confounding factors in E-R analyses for monoclonal antibodies in oncology several key determinants need to be considered. In this review we have described the importance of selecting the appropriate drug exposure metric for E-R analyses and have summarized three main approaches in oncology to address confounding in E-R analyses: $\mathrm{CPH}$ and case-matching analysis, tumor growth inhibition overall survival (TGI-OS) modeling, and clinical studies with multiple dose levels (Figure 1, Table 1 ). In addition to describing these approaches we will discuss their strengths and limitations. The current review will focus on E-R analyses for exposure-survival relationships.

\subsection{SELECTION OF DRUG EXPOSURE METRIC}

\subsubsection{Pharmacokinetic Parameters}

For monoclonal antibodies, particularly those that demonstrate time-dependent PK, the selection of the appropriate exposure metric to use for E-R analyses is critical. An E-R analysis simulation for nivolumab, for example, tested 3 different exposure metrics and resulted in different $\mathrm{E}-\mathrm{R}$ conclusions. The exposure metrics used were: (1) average concentration at steady state $\left(\mathrm{Cavg}_{\mathrm{ss}}\right),(2)$ average concentration at cycle 1 (Cavg 1 1st-dose $)$, and (3) trough concentration at cycle 1 ( Cmin $\left._{1 \text { st-dose }}\right) \cdot{ }^{12}$ When Cavg $_{\text {ss }}$ was used as the exposure metric there was a steep E-R relationship with a hazards ratio (HR) of 0.92 and 0.14 between quartiles 1 and 4, and the case-matched control arm, respectively. However, when Cavg ${ }_{1 s t-d o s e}$ and $\mathrm{Cmin}_{1 \text { st-dose }}$ were used the apparent E-R relationship was flat. The flat E-R relationship is consistent with the lack of dose-response relationship derived from the randomized dose-ranging trial for nivolumab. ${ }^{13}$ The observed inconsistency in E-R relationship between the different exposure metrics used $\left(\mathrm{Cavg}_{\mathrm{ss}}\right.$, $\mathrm{Cavg}_{1 \mathrm{st}-\mathrm{dose}}$, and $\mathrm{Cmin}_{1 \text { st-dose }}$ ) can be attributed to the dependence of $\mathrm{Cavg}_{\mathrm{ss}}$ on concentrations from later post-treatment time points. Patients with improving disease status have reduced drug clearance, so at later time points there is an apparent correlation between exposure and response. Using exposure metrics derived from earlier time points reduces the change in disease status influencing PK, and allows for more accurate assessment of the impact of treatment exposure on clinical response. ${ }^{14}$

In addition to using exposure metrics derived from early time points consideration should be placed on whether observed or model-derived exposure metrics are used in the E-R analysis. In the E-R analysis for trastuzumab emtansine (T-DM1) the results of Cox proportional-hazards modeling (CPH) were not consistent between model-derived and observed exposure metrics. After adjusting for baseline risk factors in the analyses for both metrics the model-predicted $\mathrm{Cmin}_{1 \text { st-dose }}$ and area under the curve at cycle 1 
$\left(\mathrm{AUC}_{1 \text { st-dose }}\right)$ were significantly associated with $\mathrm{OS}$ though the observed $\mathrm{Cmin}_{1 \text { st-dose }}$ and $\mathrm{AUC}_{1 \text { st-dose }}$ were not. ${ }^{15}$ Due to the limited understanding of this discrepancy a strong recommendation could not be made regarding the selection of observed or model-derived metrics, and it would be prudent for an E-R analysis to examine both. Upon examining both metrics if either of them is significantly associated with OS further investigation into the population PK model and E-R analysis is warranted.

\subsubsection{Assay Considerations}

The use of free versus total PK assays to measure drug exposure and their impact on E-R relationships should be considered. It has been suggested that since free drug concentration is in excess from binding to targets and proteins it is unsuitable for E-R analyses. ${ }^{16}$ However, free drug concentration may also reflect active drug in the circulation that can bind to targets, and therefore be relevant in an E-R analysis. It is also thought that because monoclonal antibodies are dosed in excess of target ligands total concentration would approximate free concentrations, and selection of free versus total assay would not impact the E-R analysis. ${ }^{17}$ Developing bioanalytical assays to measure free concentrations for monoclonal antibodies also faces numerous technical challenges. ${ }^{17}$ As assays are studied and developed further potential impacts on E-R analyses should be evaluated.

E-R analyses for drugs with multiple analytes such as antibody-drug conjugates (ADCs) involve additional complexities. There is a limited understanding of whether exposure to the cytotoxic drug, monoclonal antibodies, ADC, or other intermediates provides the best correlation for E-R relationships. ${ }^{18,}{ }^{19}$ The analyte driving response appears to vary across different ADCs, and this issue should be carefully considered in E-R analyses for ADCs.

\subsection{SELECTION OF ADJUSTMENT MODELS}

\subsubsection{CPH Modeling and Case Matching}

$\mathrm{CPH}$ modeling is a survival analysis in which a multivariate regression model (Equation 1) evaluates the association between covariates (e.g., baseline prognostic factors, exposure measures) and the time until a specific event occurs. The comparison of response between treatment groups is given as a HR, and this ratio is assumed to be constant over time (Equation 2). The model allows for estimation of the relationship between exposure and response. Multiple covariates can be evaluated in the model for statistical significance, and it is imperative that they are included to correct for the effects of confounding factors that might otherwise bias the E-R analysis. The structure of the model assumes that the effects of these covariates are timeindependent, and also depend upon the value of the covariate and a constant coefficient. This approach has been used to adjust for confounding factors in E-R analyses for T-DM1. ${ }^{15,20}$

$h(t)=h_{0}(t) \times \exp \left(b_{1} X_{1}+b_{2} X_{2}+\ldots b_{p} X_{p}\right)($ Equation 1$)$

where $h(t)$ describes the hazard of an event at time $t$, determined by a set of covariates $\left(X_{1}, X_{2}, \ldots, X_{p}\right.$ )$; h_{0}(t)$ describes the baseline hazard at time $t$; and the coefficients $\left(b_{1}, b_{2}, \ldots, b_{p}\right)$ describe the relationship between the covariates and the hazard.

$H R=\frac{h(t)_{y}}{h(t)_{z}}=\frac{\exp \left(b_{1} X_{1 y}+b_{2} X_{2 y}+\ldots b_{p} X_{\mathrm{py}}\right)}{\exp \left(b_{1} X_{1 z}+b_{2} X_{2 z}+\ldots b_{p} X_{\mathrm{pz}}\right)}($ Equation 2)

where $H R$ is the ratio of the expected hazards of two groups, $\mathrm{y}$ and $\mathrm{z}$, and is time-independent. Components of this equation are the same as described in Equation 1.

Case-matching analysis has been widely used in observational studies to adjust for confounding factors. The method was more recently applied to E-R analysis for the first time by Yang et al. and has since been used for E-R analyses of multiple oncology biologics such as T-DM1 and nivolumab. ${ }^{2,}$ 12, 15 Case-matching analysis adjusts for confounding factors by balancing the distribution of baseline risk factors between the control and treatment groups prior to calculating the HR. Only patients in the control arm that are similar or matched in baseline risk to patients in the treatment arm are included in the analysis. The matching process can be optimized with a variety of methods including propensity score matching, Mahalanobis distance matching, and coarsened exact matching. ${ }^{21-23}$ In the more commonly used propensity score the score is 
typically estimated using a logistic regression model, and patients with similar scores are matched. After case-matching the endpoint can be directly compared between the matched groups by a method of choice (e.g., Kaplan-Meier survival analysis, $\mathrm{CPH}$ ).

$\mathrm{CPH}$ modeling and case-matching address confounding factors in an E-R analysis by accounting for covariates in different exposure subgroups. For both approaches to successfully account for confounding in monoclonal antibodies in oncology appropriate covariates that account for imbalances in prognostic factors must be selected. The number of covariates is limited by the increasing potential for over-parameterization of results. In a comparison of response in the Q1 exposure subgroup and the control arm, Li et al . used CPH modeling and case-matching. ${ }^{15}$ Case-matching analysis demonstrated a greater reduction of the HR. While the case-matching analysis had additional covariates included that could contribute to the reduction of HR the reduction can also be attributed to the limitation of $\mathrm{CPH}$ modeling where the structure of the hazards model equation imposes assumptions about the effect of covariates on the E-R relationship.

Case-matching analysis is an appealing alternative to $\mathrm{CPH}$ modeling, as it requires no assumptions regarding the relationship between covariates and the E-R relationship. In addition, there is no specific method to select covariates used for matching, and covariates are not screened for significance in the case-matching analysis. The retention of both statistically significant and insignificant covariates may allow for an increased capacity for correction of confounding factors compared to methods that screen for covariates. Covariates that are clinically significant may be statistically insignificant in an analysis due to factors such as small sample size, variability, and correlation with other risk factors. Case-matching analysis is limited by the difficulty in matching case to control when using a small study sample or a large number of covariates. If data from an adequate sample size are available, and there is no need for validation of covariates by statistical significance case-matching appears to correct for confounding factors more effectively than $\mathrm{CPH}$ modeling.

\subsubsection{TGI-OS Modeling}

The TGI-OS model is a disease progression model. It is a useful tool in oncology to delineate E-R relationships in the presence of confounding factors. The model is composed of two parts: a TGI model that describes tumor dynamics, and a multivariate survival model that incorporates a TGI metric as a covariate on OS. The TGI metric serves as a marker of disease status. TGI-OS modeling mitigates confounding in the E$\mathrm{R}$ analysis by directly evaluating the treatment effect on TGI then separately accounting for the effect of prognostic factors on OS. By mitigating the confounding effects of prognostic factors on the relationship between treatment effect and OS, the TGI-OS model can avoid a false positive E-R relationship. ${ }^{24-26}$ The TGI model structure is typically a simple biexponential model (Equation 3 ). ${ }^{27}$

$f(t)=\exp (-d \times t)+\exp (g \times t)-1(\text { Equation } 3)^{27}$

where $f(t)$ is tumor size at time $t, d$ is the decay rate constant, and $g$ is the growth rate constant.

In multiple cancer types, the OS is correlated with the tumor dynamics such that the probability of survival decreases with the increase in tumor growth rate $\left(g\right.$ in Equation 3). ${ }^{27-37}$ Other key determinants for survival are baseline prognostic factors specific for the cancer type. Drug exposure is evaluated as a covariate in the multivariate survival model. ${ }^{24,25,29,32}$ If it is not significant this suggests a flat E-R relationship. For exposure-driven TGI models drug exposure is not evaluated as a covariate in the multivariate survival model. OS can be simulated for exposure quartiles with normalized prognostic factors to evaluate the presence of an E-R relationship. This approach can remove the confounding effects of imbalanced prognostic factors in different exposure quartiles. TGI-OS modeling has successfully evaluated E-R relationships for atezolizumab in multiple indications, and its role in E-R analysis has been increasingly accepted by regulatory agencies. $^{25,28,29,32}$

While TGI-OS modeling allows for the direct separation of treatment effect and disease effects, several limitations must be considered. Non-exposure driven TGI models while simpler and more flexible than exposure-driven models require assumptions and empirical descriptions of tumor shrinkage and growth. Model building for both exposure and non-exposure driven models requires one or more post-treatment 
assessments for tumor size, and this may not be feasible in some patients. The incorporation of multiple tumor size assessments in the model, however, makes tumor dynamics a patient-specific explanatory variable and informative predictor of survival. With the TGI-OS model, it is also difficult to account for the potential appearance of new lesions. Zecchin et al . developed a pharmacometric model to incorporate the effect of new lesions on OS in metastatic ovarian cancer, but additional examples and uses of this approach are currently limited. ${ }^{38,}{ }^{39}$ Because the TGI-OS model predicts OS based on tumor dynamics it is more suitable for use in advanced malignancies, where tumor size is typically measured over time.

\subsection{SELECTION OF STUDY DESIGN}

When an E-R analysis using an adjustment model concludes a positive E-R relationship it is difficult to discern whether there is a true positive E-R relationship, or there are additional hidden confounders. The only approach that allows for certainty in a positive E-R relationship is to study multiple dose levels of the drug in large, registrational trials. These trials should have randomized groups with balanced baseline characteristics. Studying multiple dose levels allows for the identification of a true E-R relationship because the dose-response relationship is not confounded by the prognostic factors that confound the E-R relationship. ncology phase 3 trials typically only study a single dose level, and the exposure range included in the E-R analysis for OS is limited. Including additional arms or performing separate trials to increase this exposure range allows for a more robust E-R analysis. In the previously described case of trastuzumab, the HELOISE trial studying high dose and standard dose trastuzumab revealed that the case-matching analysis conducted for the ToGA study was confounded, and that there appeared to be no causal relationship between exposure and response for trastuzumab in metastatic gastric cancer. ${ }^{2,}{ }^{3} \mathrm{~A}$ similar scenario was observed with pembrolizumab in melanoma and NSCLC. Pembrolizumab was studied across a 5 -fold dose range. Two casematching analyses were performed for patients receiving $2 \mathrm{mg} / \mathrm{kg}$ and $10 \mathrm{mg} / \mathrm{kg}$ dose levels, respectively. In the unmatched analysis a steep E-R relationship was observed across exposure ranges within each dose level. While case-matching analysis corrected this E-R relationship to a certain degree it still suggested a positive E-R relationship. When examining hazard ratios across the two dose levels, however, the apparent E-R relationship was flat and suggested that higher exposures do not increase OS. The case-matching analysis was unable to fully account for confounders. ${ }^{40}$

Because dose-response relationships are not confounded by the prognostic factors that confound E-R relationships studying multiple dose levels is a robust approach to examine E-R relationships. Unlike statistical approaches discussed in the previous sections it requires no assumptions about covariates or the structure of the E-R relationship. The major limitation with this approach is the time and cost associated with additional trials or treatment arms. In addition, this approach may not be feasible in rare populations. The utility of studying multiple dose levels may also depend on characteristics of the drug. If a drug has a wide therapeutic window (i.e. monoclonal antibodies), and tested doses appear to be at the top of the dose-response curve studying multiple dose levels in registrational trials may not be necessary. It may be useful if a drug has a narrow therapeutic window and requires quantification of E-R relationships for optimal dose selection. Ultimately, limitations in feasibility motivate sponsors to consider alternative approaches to E-R analyses before studying multiple dose levels.

\section{DISCUSSION}

The inability to select an appropriate dose in pivotal trials has been shown to contribute to the declining success rates of drug development programs. ${ }^{41,42}$ A study examining FDA approval packages between 2015 and 2017 found that in a third of development programs no E-R analysis was reported. ${ }^{43}$ The expanded use of E-R analysis in more drug development programs may serve as a solution for declining success rates. E-R analysis is particularly useful in early clinical trials where multiple doses are administered to inform dose selection and optimization. It is often repeated in phase 3 trials given the meaningful sample size for efficacy and safety interpretation. Rather than assuming one dose fits all patients this approach identifies whether specific patient subgroups would benefit from alternative doses. A successful example of E-R application was shown for the exposure-survival analysis of ipilimumab. Ipilimumab was originally approved in several countries at a dose of $3 \mathrm{mg} / \mathrm{kg}$ for the treatment of advanced melanoma. A phase 2 dose-ranging study, 
however, suggested improvement in OS with the $10 \mathrm{mg} / \mathrm{kg}$ dose. ${ }^{44}$ While this study was not statistically powered to detect differences in survival an E-R analysis pooling data from four phase 2 trials demonstrated that OS improved with increasing exposure. In the CPH model results patients in the $5^{\text {th }}$ and $95^{\text {th }}$ percentiles of steady state trough concentration $\left(\mathrm{Cmin}_{\mathrm{ss}}\right)$ had an OS HR of 1.52 and 0.552 , respectively, relative to patients with median $\mathrm{Cmin}_{\mathrm{ss}} .{ }^{45}$ This suggested that OS improved with increased ipilimumab doses. In the post-marketing trial conducted with the $3 \mathrm{mg} / \mathrm{kg}$ and $10 \mathrm{mg} / \mathrm{kg}$ doses this relationship was confirmed. Median OS was 15.7 months for the $10 \mathrm{mg} / \mathrm{kg}$ dose group, and 11.5 months for the $3 \mathrm{mg} / \mathrm{kg}$ dose group (HR $0.84, \mathrm{p}=0.04) .{ }^{46}$ The results of this phase 3 trial are included in the ipilimumab label, and demonstrate that E-R analyses could identify potential survival benefits gained from increased doses and exposures. While the $10 \mathrm{mg} / \mathrm{kg}$ dose provided a survival benefit it was also associated with increased treatment-related adverse events. The $3 \mathrm{mg} / \mathrm{kg}$ dose was selected as the labeled dose after accounting for efficacy benefit and safety risk.

While the utility of E-R analyses applies across a variety of therapeutic areas additional considerations are needed for oncology due to the impact of prognostic factors on both exposure and outcome. Performance status, clinical symptoms (dyspnea, appetite loss, cognitive function), primary tumor site, and c-reactive protein $(\mathrm{CRP})$ concentration are examples of prognostic factors used to predict outcome in clinical settings. ${ }^{47,} 48$ In oncology, E-R is more than just considering the unidirectional relationship where the dose affects exposure which subsequently affects response. OS is often the primary response endpoint for oncology trials, and its relationship with exposure is confounded by prognostic factors. Recognizing and accounting for the impact of time-varying clinical response and prognostic factors on exposure are critical for accurate E-R interpretations. ${ }^{49}$ This relationship is illustrated by the findings for nivolumab, avelumab, durvalumab, and pembrolizumab where patients with improved post-treatment disease status showed greater time-dependent decreases in drug CL. ${ }^{7-10}$ The mechanism is not fully understood, but there is an interaction between clinical response, prognostic factors, and exposure. When patients respond to treatment their prognostic factors improve, which in turn decrease the drug CL and increase drug exposure (Figure 2). In an E-R analysis this could lead to incorrect conclusions that higher drug exposure caused clinical response when in fact the E- $R$ relationship is confounded by the effect of changing prognostic factors on exposure. This may be caused by the unique nature of disease progression in oncology. As a patient's disease status declines clinical changes such as cachexia and inflammation can increase the catabolism and clearance of both endogenous and therapeutic proteins. ${ }^{50,}{ }^{51}$ This is supported by the significance of tumor burden and albumin as a covariate on CL in the population PK analyses for nivolumab, avelumab, durvalumab, and pembrolizumab. ${ }^{8-10}$, 12 Clearance increased with higher tumor burden and lower albumin concentrations. In addition, time-dependent PK was observed for nivolumab in advanced malignancies, but not in patients with resected melanoma. ${ }^{52}$ The latter had tumors surgically resected prior to adjuvant treatment with nivolumab and were overall healthier than patients with advanced malignancies. This further supports the impact of disease status and prognostic factors on exposure. Looking prospectively, these collective observations also suggest that the presence of time-dependent PK, and the significance of albumin as a covariate on CL would indicate the risk of a confounded E-R analysis.

A confounded E-R analysis may result in false positive E-R relationships which may lead to the wrong conclusion that the dose for patients with lower exposure is suboptimal. As seen in the ToGA/HELOISE example it may lead to the initiation of a new trial in an attempt to rescue patients who failed treatment. Considering these risks three mitigation strategies have been in this review: $\mathrm{CPH}$ modeling and case matching analysis, TGI-OS modeling, and multiple dose study design. Studying multiple dose levels in randomized, balanced groups appears to be an effective approach that can distinguish with certainty between a true positive E-R relationship versus a false positive relationship with hidden confounders. This strategy, however, is impractical in most oncology indications, and may offer limited value for monoclonal antibodies with a wide therapeutic window. TGI-OS modeling explicitly separates the drug-specific and disease-specific effect on OS when evaluating the E-R relationship. It also incorporates an estimate of tumor dynamics which serves as an informative biomarker of disease status. CPH modeling and case-matching analysis lack this separation between drug and disease-specific effects. This makes it more challenging to consistently 
distinguish between exposure- and prognostic factor-driven changes in OS. Case-matching may be preferred over $\mathrm{CPH}$ modeling due to the assumptions involved in $\mathrm{CPH}$ modeling regarding the relationship between predictors and outcome. While a suggestion of the relative utility of each approach is made here the unique limitations of each methodology should be considered.

While the effect of changing prognostic factors on exposure and OS can confound exposure-efficacy relationships it does not appear to significantly impact exposure-safety relationships. Among molecules discussed here it appears that exposure-safety analyses for pembrolizumab, nivolumab, durvalumab, and T-DM1 have not faced the same confounding issues as exposure-efficacy analyses. ${ }^{15,} 20,53-58$ If patients with worsening disease status and prognostic factors are more likely to experience adverse events, and have a decreased drug exposure it could be thought that differences in prognostic factors can confound the exposure-safety relationship. The confounding, however, would contribute to an inverse E-R relationship rather than a positive relationship. In addition, because safety endpoints in E-R analyses are usually drug-related adverse events rather than disease-related adverse events exposure-safety relationships may be less likely to be influenced by differences in prognostic factors.

Current oncology drug development is rapid and aggressive. Many recent development programs bypass a dose-ranging phase 2 trial and go directly from phase 1 to phase 3 trials with a single dose level. In some programs, a phase 2 trial is done, but only with a single dose level or a limited efficacy endpoint. This severely limits the range of exposure data available for an exposure-survival analysis and increases the risk of a confounded E-R analysis. The FDA's E-R Guidance has previously described the risk of characterizing E-R relationships based on data from single dose levels. ${ }^{59}$ Sponsors should consider conducting expanded dose-ranging trials early in development programs to better inform dose selection and potentially avoid the need to study multiple dose levels in late phase trials. Despite the current limitations of E-R analyses they are required to be included as part of a filing package. The HELOISE trial is an example of the potential risks of confounded E-R analyses. The E-R analysis performed using data from the ToGA trial supported the conduct of the HELOISE trial. No dose-response relationship was observed, however, and patients did not benefit from higher doses of trastuzumab. If the E-R analysis is limited by the range of available exposure data, and could be confounded any observed E-R relationship should be interpreted with great caution.

Drug development must not only focus on developing novel treatment modalities, but also on selecting the optimal dose for patients. E-R analysis is a useful tool for dose optimization in a variety of therapeutic areas, and also has many applications to support modern drug development. Despite its wide utility E-R analysis in oncology faces unique challenges when applied to monoclonal antibodies tested at a single dose level. E-R analyses in oncology are susceptible to confounding from unique, disease-related factors. Mitigation strategies presented in the current paper can be employed to account for confounding factors and elucidate the true E-R relationship. In a broader scope, the design of oncology drug development programs may be structured to more effectively inform dose-response and E-R relationships for dose optimization. Once an E-R analysis is performed its application in decision-making must be carefully considered based on the methodology and the data used in the analysis. The improvement and effective use of E-R analysis is an effort that must be addressed on multiple fronts of oncology drug development with the common goal of maximizing benefit to the patient and minimizing toxicities.

\section{COMPETING INTERESTS}

The authors except SK are all employees of Genentech, Inc., and stockholders of the Roche Group. SK has no conflict of interest to declare.

\section{REFERENCES:}

1. Minchella K, Xu H, Al-Huniti N. Exposure-response methods and dose approval of new oncology drugs by FDA from 2005 to 2015. J. of Clin. Oncol. 2016; 34: 2530-30.

2. Yang J, Zhao H, Garnett C, et al. The combination of exposure-response and case-control analyses in regulatory decision making. J Clin Pharmacol. 2013; 53: 160-6. 
3. Shah MA, Xu RH, Bang YJ, et al. HELOISE: Phase IIIb Randomized Multicenter Study Comparing Standard-of-Care and Higher-Dose Trastuzumab Regimens Combined With Chemotherapy as First-Line Therapy in Patients With Human Epidermal Growth Factor Receptor 2-Positive Metastatic Gastric or Gastroesophageal Junction Adenocarcinoma. J Clin Oncol. 2017; 35: 2558-67.

4. Li J, Levi M, Charoin J-E, et al. Rituximab Exhibits a Long Half-Life Based on a Population Pharmacokinetic Analysis in Non-Hodgkin's Lymphoma (NHL) Patients. Blood 2007; 100.

5. Li J, Zhi J, Wenger M, et al. Population pharmacokinetics of rituximab in patients with chronic lymphocytic leukemia. J Clin Pharmacol. 2012; 52: 1918-26.

6. Rozman S, Grabnar I, Novakovic S, Mrhar A, Jezersek Novakovic B. Population pharmacokinetics of rituximab in patients with diffuse large B-cell lymphoma and association with clinical outcome. $\mathrm{Br} J$ Clin Pharmacol. 2017; 83: 1782-90.

7. Bajaj G, Wang X, Agrawal S, Gupta M, Roy A, Feng Y. Model-Based Population Pharmacokinetic Analysis of Nivolumab in Patients With Solid Tumors. CPT Pharmacometrics Syst Pharmacol. 2017; 6: 58-66.

8. Baverel PG, Dubois VFS, Jin CY, et al. Population Pharmacokinetics of Durvalumab in Cancer Patients and Association With Longitudinal Biomarkers of Disease Status. Clin Pharmacol Ther. 2018; 103: 631-42.

9. Li H, Yu J, Liu C, et al. Time dependent pharmacokinetics of pembrolizumab in patients with solid tumor and its correlation with best overall response. J Pharmacokinet Pharmacodyn. 2017; 44: 403-14.

10. Wilkins JJ, Brockhaus B, Dai H, et al. Time-Varying Clearance and Impact of Disease State on the Pharmacokinetics of Avelumab in Merkel Cell Carcinoma and Urothelial Carcinoma. CPT Pharmacometrics Syst Pharmacol. 2019; 8: 415-27.

11. Ryman JT, Meibohm B. Pharmacokinetics of Monoclonal Antibodies.CPT Pharmacometrics Syst Pharmacol. 2017; 6: 576-88.

12. Liu C, Yu J, Li H, et al. Association of time-varying clearance of nivolumab with disease dynamics and its implications on exposure response analysis. Clin Pharmacol Ther . 2017; 101: 657-66.

13. EMA. Assessment Report. OPDIVO. International non-proprietary name: Nivolumab. https://www.ema.europa.eu/en/documents/variation-report/opdivo-h-c-3985-ii-0008-epar-scientific-

discussion-variation_en.pdf. In, 2016.

14. Wang Y, Booth B, Rahman A, Kim G, Huang SM, Zineh I. Toward greater insights on pharmacokinetics and exposure-response relationships for therapeutic biologics in oncology drug development. Clin Pharmacol Ther. 2017; 101: 582-84.

15. Li C, Wang B, Chen SC, et al. Exposure-response analyses of trastuzumab emtansine in patients with HER2-positive advanced breast cancer previously treated with trastuzumab and a taxane. Cancer Chemother Pharmacol. 2017; 80: 1079-90.

16. Imamura CK. Therapeutic drug monitoring of monoclonal antibodies: Applicability based on their pharmacokinetic properties. Drug Metab Pharmacokinet. 2019; 34: 14-18.

17. Lee JW, Kelley M, King LE, et al. Bioanalytical approaches to quantify "total" and "free" therapeutic antibodies and their targets: technical challenges and PK/PD applications over the course of drug development. AAPS J 2011; 13: 99-110.

18. Gorovits B, Alley SC, Bilic S, et al. Bioanalysis of antibody-drug conjugates: American Association of Pharmaceutical Scientists Antibody-Drug Conjugate Working Group position paper. Bioanalysis2013; 5: 997-1006. 
19. Kraynov E, Kamath AV, Walles M, et al. Current Approaches for Absorption, Distribution, Metabolism, and Excretion Characterization of Antibody-Drug Conjugates: An Industry White Paper. Drug Metab Dispos. 2016; 44: 617-23.

20. Wang J, Song P, Schrieber S, et al. Exposure-response relationship of T-DM1: insight into dose optimization for patients with HER2-positive metastatic breast cancer. Clin Pharmacol Ther. 2014; 95: $558-64$.

21. Iacus SM, King G, Porro G. Multivariate Matching Methods That are Monotonic Imbalance Bounding. J. Am. Stat. Assoc. 2011; 106: 345-61.

22. Rosenbaum PR, Rubin DB. The central role of the propensity score in observational studies for causal effects. Biometrika 1983; 70: 41-55.

23. Rubin DB. Inference and missing data. Biometrika 1976; 63: 581-92.

24. Han K, Chanu P, Jonsson F, et al. Exposure-Response and Tumor Growth Inhibition Analyses of the Monovalent Anti-c-MET Antibody Onartuzumab (MetMAb) in the Second- and Third-Line Non-Small Cell Lung Cancer.AAPS J. 2017; 19: 527-33.

25. Morrissey KM, Marchand M, Patel H, et al. Alternative dosing regimens for atezolizumab: an example of model-informed drug development in the postmarketing setting. Cancer Chemother Pharmacol. 2019; 84: 1257-67.

26. Quartino AL, Claret L, Li J, et al. Evaluation of tumor size metrics to predict survival in advanced gastric cancer. PAGE 2013; 22: Abstr 2812.

27. Stein WD, Figg WD, Dahut W, et al. Tumor growth rates derived from data for patients in a clinical trial correlate strongly with patient survival: a novel strategy for evaluation of clinical trial data. Oncologist 2008; 13: 1046-54.

28. Bruno R, Claret L, Jin YJ, Girish S. FDA-ISoP public workshop: model informed drug development (MIDD) for oncology products. Silver Spring,MD: FDA. 2018 Feb 1; Available from: https://www.fda.gov/drugs/news-events-human-drugs/fda-isop-public-workshop-modelinformed-drug-development-midd-oncology-products. In, 2018.

29. Bruno R, Claret L, Wu B, et al. A tumor growth rate/overall survival model for atezolizumab as an early predictor of OS in patients with first or second line metastatic urothelial carcinoma. J. Clin. Oncol. 2018; 36: 62-62.

30. Chigutsa E, Long AJ, Wallin JE. Exposure-Response Analysis of Necitumumab Efficacy in Squamous Non-Small Cell Lung Cancer Patients. CPT Pharmacometrics Syst Pharmacol. 2017; 6: 560-68.

31. Claret L, Gupta M, Han K, et al. Evaluation of tumor-size response metrics to predict overall survival in Western and Chinese patients with first-line metastatic colorectal cancer. J Clin Oncol. 2013; 31: 2110-4.

32. Claret L, Jin JY, Ferte C, et al. A Model of Overall Survival Predicts Treatment Outcomes with Atezolizumab versus Chemotherapy in Non-Small Cell Lung Cancer Based on Early Tumor Kinetics. Clin Cancer Res. 2018; 24: 3292-98.

33. Claret L, Lu JF, Bruno R, Hsu CP, Hei YJ, Sun YN. Simulations using a drug-disease modeling framework and phase II data predict phase III survival outcome in first-line non-small-cell lung cancer. Clin Pharmacol Ther. 2012; 92: 631-4.

34. Feng Y, Wang X, Suryawanshi S, Bello A, Roy A. Linking Tumor Growth Dynamics to Survival in Ipilimumab-Treated Patients With Advanced Melanoma Using Mixture Tumor Growth Dynamic Modeling. CPT Pharmacometrics Syst Pharmacol. 2019; 8: 825-34. 
35. Tardivon C, Desmee S, Kerioui M, et al. Association Between Tumor Size Kinetics and Survival in Patients With Urothelial Carcinoma Treated With Atezolizumab: Implication for Patient Follow-Up. Clin Pharmacol Ther. 2019; 106: 810-20.

36. Wang Y, Sung C, Dartois C, et al. Elucidation of relationship between tumor size and survival in nonsmall-cell lung cancer patients can aid early decision making in clinical drug development. Clin Pharmacol Ther. 2009; 86: 167-74.

37. Zheng Y, Narwal R, Jin C, et al. Population Modeling of Tumor Kinetics and Overall Survival to Identify Prognostic and Predictive Biomarkers of Efficacy for Durvalumab in Patients With Urothelial Carcinoma. Clin Pharmacol Ther. 2018; 103: 643-52.

38. Bruno R, Bottino D, de Alwis DP, et al. Progress and Opportunities to Advance Clinical Cancer Therapeutics Using Tumor Dynamic Models. Clin Cancer Res. 2020; 26: 1787-95.

39. Zecchin C, Gueorguieva I, Enas NH, Friberg LE. Models for change in tumour size, appearance of new lesions and survival probability in patients with advanced epithelial ovarian cancer. $\mathrm{Br} J$ Clin Pharmacol. 2016; 82: 717-27.

40. Turner DC, Kondic AG, Anderson KM, et al. Pembrolizumab Exposure-Response Assessments Challenged by Association of Cancer Cachexia and Catabolic Clearance. Clin Cancer Res. 2018; 24: 5841-49.

41. Bornkamp B, Bretz F, Dmitrienko A, et al. Innovative approaches for designing and analyzing adaptive dose-ranging trials. J Biopharm Stat. 2007; 17: 965-95.

42. Hay M, Thomas DW, Craighead JL, Economides C, Rosenthal J. Clinical development success rates for investigational drugs. Nat Biotechnol. 2014; 32: 40-51.

43. Lyauk YK, Jonker DM, Lund TM. Dose Finding in the Clinical Development of 60 US Food and Drug Administration-Approved Drugs Compared With Learning vs. Confirming Recommendations. Clin Transl Sci. 2019; 12: 481-89.

44. Wolchok JD, Neyns B, Linette G, et al. Ipilimumab monotherapy in patients with pretreated advanced melanoma: a randomised, double-blind, multicentre, phase 2, dose-ranging study. Lancet Oncol. 2010; 11: $155-64$.

45. Feng Y, Roy A, Masson E, Chen TT, Humphrey R, Weber JS. Exposure-response relationships of the efficacy and safety of ipilimumab in patients with advanced melanoma. Clin Cancer Res. 2013; 19: 3977-86.

46. Ascierto PA, Del Vecchio M, Robert C, et al. Ipilimumab $10 \mathrm{mg} / \mathrm{kg}$ versus ipilimumab $3 \mathrm{mg} / \mathrm{kg}$ in patients with unresectable or metastatic melanoma: a randomised, double-blind, multicentre, phase 3 trial.Lancet Oncol. 2017; 18: 611-22.

47. Gripp S, Moeller S, Bolke E, et al. Survival prediction in terminally ill cancer patients by clinical estimates, laboratory tests, and self-rated anxiety and depression. J Clin Oncol. 2007; 25: 3313-20.

48. Maltoni M, Caraceni A, Brunelli C, et al. Prognostic factors in advanced cancer patients: evidence-based clinical recommendations-a study by the Steering Committee of the European Association for Palliative Care. J Clin Oncol. 2005; 23: 6240-8.

49. Dai HI, Vugmeyster Y, Mangal N. Characterizing Exposure-Response Relationship for Therapeutic Monoclonal Antibodies in Immuno-Oncology and Beyond: Challenges, Perspectives, and Prospects. Clin Pharmacol Ther. 2020.

50. McMillan DC. An inflammation-based prognostic score and its role in the nutrition-based management of patients with cancer. Proc Nutr Soc. 2008; 67: 257-62.

51. Trobec K, Kerec Kos M, von Haehling S, Springer J, Anker SD, Lainscak M. Pharmacokinetics of drugs in cachectic patients: a systematic review. PLoS One 2013; 8: e79603. 
52. Hamuro L, Statkevich P, Bello A, Roy A, Bajaj G. Nivolumab Clearance Is Stationary in Patients With Resected Melanoma on Adjuvant Therapy: Implications of Disease Status on Time-Varying Clearance. Clin Pharmacol Ther. 2019; 106: 1018-27.

53. Chatterjee M, Turner DC, Felip E, et al. Systematic evaluation of pembrolizumab dosing in patients with advanced non-small-cell lung cancer. Ann Oncol. 2016; 27: 1291-8.

54. FDA. Clinical pharmacology and biopharmaceutics review: durvalumab. https://www.accessdata.fda.gov/drugsatfda_docs/nda/2017/761069Orig1s000TOC.cfm. In, 2017.

55. Jin C, Zheng Y, Jin X, et al. Exposure-efficacy and safety analysis of durvalumab in patients with urothelial carcinoma (UC) and other solid tumors. J. of Clin. Oncol. 2017; 35: 2568-68.

56. Jin J, Wang B, Gao Y, et al. Exposure-safety relationship of trastuzumab emtansine (T-DM1) in patients with HER2-positive locally advanced or metastatic breast cancer (MBC). J. of Clin. Oncol.2013; 31: $646-46$.

57. Kang SP, Chatterjee M, Ahamadi M, et al. 3344 Relationship between pembrolizumab exposure and efficacy/safety in 1016 patients (pts) with advanced or metastatic melanoma. Eur $J$ of Cancer. 2015; 51: S682.

58. Wang X, Feng Y, Bajaj G, et al. Quantitative Characterization of the Exposure-Response Relationship for Cancer Immunotherapy: A Case Study of Nivolumab in Patients With Advanced Melanoma. CPT Pharmacometrics Syst Pharmacol. 2017; 6: 40-48.

59. FDA. Guidance Document. Exposure-Response Relationships — Study Design, Data Analysis, and Regulatory Applications May 2003. http://www.fda.gov/downloads/drugs guidancecomplianceregulatoryinformation/guidances/ucm072109.pdf. In, 2018.

\section{Hosted file}

Kawakatsu ER Review Table 1.docx available at https://authorea.com/users/351708/articles/ 476231-confounding-factors-in-exposure-response-analyses-and-mitigation-strategies-formonoclonal-antibodies-in-oncology

\section{Hosted file}

Kawakatsu ER Review Figure Legends.docx available at https://authorea.com/users/351708/ articles/476231-confounding-factors-in-exposure-response-analyses-and-mitigationstrategies-for-monoclonal-antibodies-in-oncology

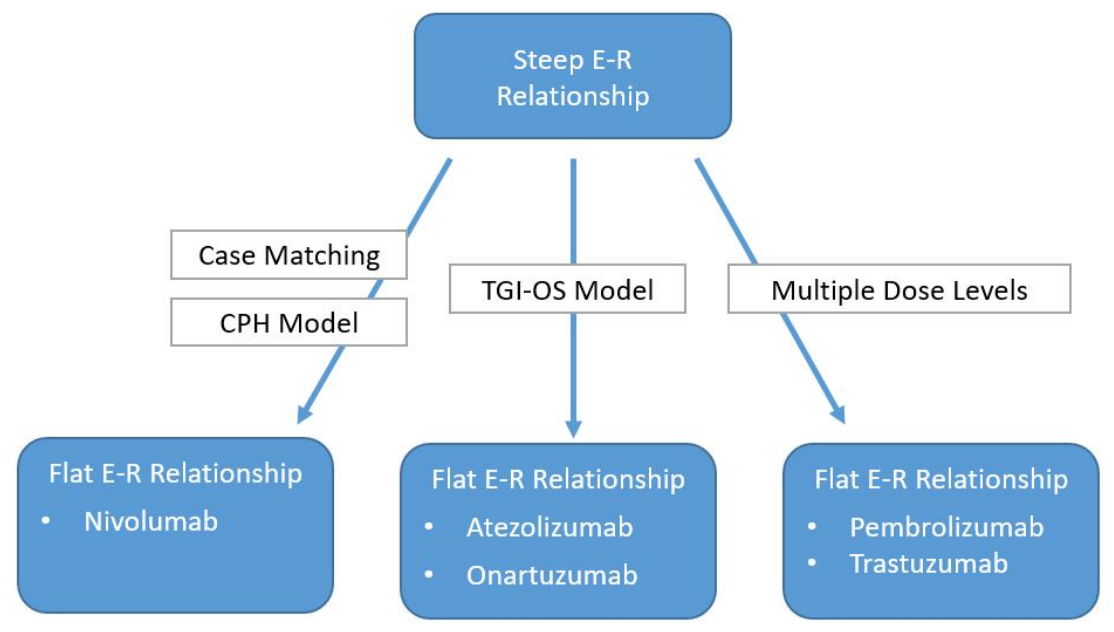




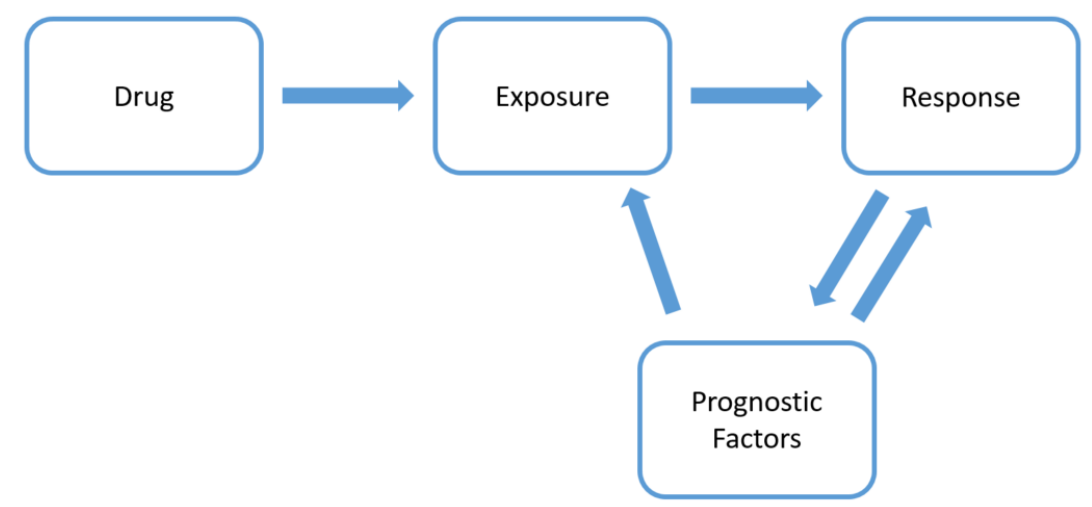

